\title{
Biomassa cítrica e fungicidas combinados com desfolha no controle da Sigatoka Amarela em banana "Prata"
}

Citric biomass and fungicides associated to leaf removal in Yellow Sigatoka disease control for banana "Prata"

\author{
Luiz Augusto Martins Peruch ${ }^{1 *}$, André Martins Medeiros ${ }^{2}$ e Celso Lopes de Albuquerque Junior ${ }^{2}$
}

Recebido em 12/12/2014 / Aceito em 02/09/2015

\section{RESUMO}

A sigatoka amarela (Mycosphaerella musicola) ainda é a principal doença da cultura da bananeira no estado de Santa Catarina. Este trabalho teve como objetivo avaliar a eficiência de fungicidas e da desfolha no controle da sigatoka em banana 'Prata'. Foram testados seis tratamentos, combinando-se três formulações (Tebuconazole $0,5 \mathrm{~L} \mathrm{ha}^{-1}$ - tiofanato metílico $0,6 \mathrm{~L}$ ha ${ }^{-1}$; Biomassa cítrica $1,0 \mathrm{~L} \mathrm{ha}^{-1}$; água) com ou sem desfolha. As variáveis avaliadas foram área abaixo da curva de progresso (AACPD) e soma bruta final (SBfinal) da doença. O delineamento experimental foi blocos ao acaso em esquema fatorial com cinco repetições, comparados entre si pelo teste de Duncan $(\mathrm{P}<0,05)$. Foram verificadas diferenças significativas para as formulações, mas não para desfolha, em comparação com testemunha. Para o fator formulação verificou-se que os fungicidas e a biomassa cítrica proporcionaram reduções significativas da área abaixo da curva de progresso da doença (AACPD) e soma bruta final (SBfinal) em comparação com testemunha. Os fungicidas e a biomassa cítrica reduziram a área abaixo da curva de progresso em 65 e $63 \%$, assim como a soma bruta final caiu 83 e $73 \%$ em comparação com testemunha, respectivamente. Não se verificou influência da desfolha no controle da sigatoka, porém outros estudos com aplicações mais frequentes devem ser realizados para quantificar melhor seu efeito. Concluiu-se que os fungicidas e a biomassa cítrica são eficientes no controle da sigatoka.

PALAVRAS-CHAVE: Mycosphaerella musicola, controle químico, controle cultural.

\section{ABSTRACT}

Yellow sigatoka (Mycosphaerella musicola) is the main disease in banana areas in the Santa Catarina state. The objective of this research was to evaluate the effect of different methods of chemical and cultural control of sigatoka disease on Prata banana in subtropical climate. Six treatments were tested, evaluating three formulations (Tebuconazole $0,5 \mathrm{~L}$ $\mathrm{ha}^{-1}$ - Tiofanato metílico $0,6 \mathrm{~L} \mathrm{ha}^{-1}$; Citric biomass $1,0 \mathrm{~L} \mathrm{ha}^{-1}$; water) with or without leaf removal. The variables evaluated were under the curve of progress (AUDPC) and final gross sum (GSfinal) area. The experimental design was randomized blocks in factorial scheme with five repetitions. Treatments were compared with Duncan's test $(\mathrm{P}<0,05)$. Significant differences were verified for products compared to control, but not for leaf removal. It was found that fungicides and citric biomass resulted in significant reductions in AUDPC and GS final when compared to control. Fungicides and citric biomass reduced area under disease progress curve by 65 and $63 \%$, as well as final gross sum were 87 and $73 \%$, respectively. Results showed small influence of leaf removal, but new studies should evaluate its influence with higher frequency of defoliation. Fungicides and citric biomass are efficient in controlling sigatoka.

KEYWORDS: Mycosphaerella musicola, chemical control, cultural control.

\footnotetext{
${ }^{1}$ Empresa de Pesquisa Agropecuária e Extensão Rural de Santa Catarina, Urussanga, SC, Brasil.

${ }^{2}$ Universidade do Sul de Santa Catarina, Tubarão, SC, Brasil.

*Autor para correspondência <lamperuch@epagri.sc.gov.br>.
} 


\section{INTRODUÇÃO}

No Estado de Santa Catarina, a banana é a principal fruta cultivada, ocupando cerca de 30.000 hectares e produzindo anualmente cerca de 650.000 toneladas. Em termos sociais, esta atividade tem grande importância para o Estado, pois cerca de 25.000 produtores exploram a cultura. No litoral Sul Catarinense, a cultura ocupa 8.500 hectares, sendo a segunda maior região produtora do Estado (SÔNEGO et al. 2003). Estima-se que $80 \%$ dos bananais da região são formados pelas cultivares Branca e Enxerto, pertencentes ao subgrupo Prata, que apresentam maior adaptação ao clima local.

A broca-da-bananeira, tripes, mal-do-panamá e o mal-de-sigatoka podem ser considerados os principais problemas fitossanitários da cultura. Em Santa Catarina ocorrem a sigatoka amarela (Mycosphaerella musicola), e mais recentemente, foi detectada a sigatoka negra ( $M$. fijiensis) (LICTHEMBERG et al. 2010). Entretanto, segundo PERUCH et al. (2010) a sigatoka amarela ainda prevalece nas condições do litoral Sul Catarinense. Estas doenças causam a destruição das folhas, reduzindo a área fotossintética e provocando queda de produção (CHILLET et al. 2009).

Pulverizações com fungicidas, desfolha e cirurgia de folhas doentes, manejo das plantas espontâneas, cultivares resistentes e condução correta do bananal são métodos de controle recomendados para o manejo da doença (MARIN et al. 2003, LAPEYRE DE BELLAIRE et al. 2010).

$\mathrm{O}$ controle químico pelo uso de fungicidas sintéticos é uma das principais práticas de manejo da sigatoka. Um aspecto fundamental no sucesso no controle químico é o manejo da resistência. Em países com alta pressão da sigatoka negra têm-se relatado a detecção da resistência pelo patógeno para diferentes princípios ativos de fungicidas sistêmicos (AMIL et al. 2007, BRENT \& HOLOMON 2007). Por este motivo é importante avaliar a eficiência de estratégias distintas de controle para diminuir o problema de resistência dos fungicidas em relação à sigatoka.

Fungicidas alternativos formulados com bactérias, ácido salicílico e extratos vegetais são novidades recentes no controle da sigatoka. Fungicidas a base de Bacillus subtilis e B. pumilus foram registrados para controle da sigatoka negra (ALVINDIA 2012) em vários países da America do Sul. Testes com ativadores de resistência também têm demonstrado potencial para controle da doença. Segundo VAWDREY \& GRICE (2005), o ácido salicílico (Bion) associado ao mancozeb propiciou controle adequado da sigatoka amarela. Formulações de biomassa cítrica também têm mostrado potencial no controle da doença pelo seu efeito sanitizante e de indução de resistência (HANADA et al. 2004), mas faltam estudos a campo.

A desfolha sanitária é apontada como uma importante prática de controle da sigatoka (MARÍN et al. 2003). Esta consiste na eliminação de folhas secas, senescentes, doentes e quebradas. Alguns trabalhos têm apontado para importância dessa prática no manejo da sigatoka, especialmente quando combinados com outros métodos de controle (JIMENEZ et al. 2007).

Poucos estudos de controle da sigatoka têm sido conduzidos em clima subtropical sob condições de campo. Dados relacionando a eficiência de produtos e de práticas culturais são raros na literatura. O objetivo deste trabalho foi avaliar o efeito da biomassa cítrica e fungicidas combinados com desfolha no controle da sigatoka amarela em bananeira 'Prata' no Estado de Santa Catarina.

\section{MATERIAL E MÉTODOS}

O experimento foi instalado num cultivo comercial de banana Enxerto em Jacinto Machado, estado de Santa Catarina, no período de agosto a novembro de 2007. O clima da região é Subtropical Úmido com Verão Quente (Cfa), segundo a classificação de Köeppen. A temperatura média anual é de $19,4{ }^{\circ} \mathrm{C}$, oscilando de $14,6^{\circ} \mathrm{C}$ em junho a $24,1^{\circ} \mathrm{C}$ em fevereiro. A precipitação total anual é de $1.624 \mathrm{~mm}$ com chuvas bem distribuídas ao longo do ano e sem estação seca definida. A umidade relativa do ar média anual é de $80 \%$, oscilando de $76 \%$ em dezembro a $83 \%$ em junho, com frequente formação de orvalho, principalmente no outono, inverno e primavera.

Foram testados seis tratamentos, associando-se três formulações com ou sem a prática da desfolha: T1) testemunha, T2) limpeza de folhas, T3) tebuconazole $\left(0,5 \mathrm{Lha}^{-1}\right) /$ tiofanato metílico $\left(0,6 \mathrm{~L} \mathrm{ha}^{-1}\right)$ sem desfolha, T4) tebuconazole $\left(0,5 \mathrm{~L} \mathrm{ha}^{-1}\right) /$ tiofanato metílico $(0,6$ $\left.\mathrm{L} \mathrm{ha}^{-1}\right)$ com desfolha, T5) biomassa cítrica $\left(1,0 \mathrm{~L} \mathrm{ha}^{-1}\right)$ sem desfolha, T6) biomassa cítrica $\left(1,0 \mathrm{~L} \mathrm{ha}^{-1}\right)$ com desfolha. Nas aplicações dos fungicidas alternaram-se as formulações tebuconazole com tiofanato metílico em cada pulverização. Os tratamentos culturais e químicos foram efetuados com intervalos de 30 
dias. As desfolhas foram efetuadas nas folhas com severidade superior a $30 \%$, antes das pulverizações dos fungicidas e da biomassa cítrica. O equipamento utilizado nas pulverizações foi atomizador costal com bico verde, volume de calda de $20 \mathrm{~L} \mathrm{ha}^{-1}$. A calda foi preparada pela mistura das formulações em óleo mineral, seguido da adição de água na proporção de $1: 1$.

Acondução do bananal seguiu as recomendações técnicas da cultura, exceto pelos aspectos manejados no experimento (ROBINSON 2006). As famílias foram conduzidas no sistema mãe-filha-neta, sendo efetuadas desbrotas quando necessário. As adubações foram efetuadas em função da análise de solo. $\mathrm{O}$ manejo das plantas espontâneas foi realizado por roçadas manuais e coroamento das bananeiras com capina manual.

O efeito dos tratamentos sobre a doença foi determinado pela avaliação semanal da soma bruta das lesões nas folhas 2, 3 e 4 em duas plantas marcadas por parcela, conforme o método de Pré-aviso bioclimático. Os dados de soma bruta foram utilizados para calcular a área abaixo da curva de progresso da doença (AACPD) através da expressão: AACPD $=\Sigma$ $\left(y_{\mathrm{i}}+y_{\mathrm{i}+1}\right) / 2 . d_{\mathrm{ti}}$ onde $y_{\mathrm{i}}$ e $\mathrm{y}_{\mathrm{i}+1}$ são os valores de severidade observados em duas avaliações consecutivas e $d_{\mathrm{ti}} \mathrm{o}$ intervalo entre as avaliações. Os dados de soma bruta da sexta avaliação foram utilizados para determinar a variável soma bruta final (SBfinal). Os dados de soma bruta também foram utilizados para confeccionar as curvas de progresso da doença.

O delineamento experimental adotado foi blocos casualizados em arranjo fatorial com seis tratamentos, sendo dois fatores e três níveis, e cinco repetições, perfazendo 30 parcelas. As parcelas ocupavam 90 $\mathrm{m}^{2}$, considerando as plantas das extremidades como bordaduras. As variáveis de AACPD e SBfinal foram submetidas à análise de variância e ao teste de médias de Duncan ao nível de 5\% de probabilidade de erro. Os dados foram transformados para raiz (x) a fim de atender os pressupostos da análise da variância. As análises estatísticas foram realizadas pelo programa SAEG (RIBEIRO JUNIOR 2001).

\section{RESULTADOS E DISCUSSÃO}

Em relação à sanidade das plantas, avaliada pela área abaixo da curva de progresso da doença (AACPD), foram verificadas diferenças significativas entre as plantas tratadas com formulações e a testemunha (Tabela 1). Para desfolha não foram verificadas diferenças significativas entre os tratamentos. Também não foram verificadas diferenças significativas para as interações entre formulações e desfolha das plantas para as variáveis (Tabela 1).

Pela análise da AACPD verificou-se que as formulações propiciaram controle da sigatoka. Foram observadas reduções de cerca de 65 e $63 \%$ para biomassa cítrica e fungicidas comparados com a testemunha, respectivamente (Tabela 2). Os valores similares das médias dessa variável reforçam o fato de não se verificarem diferenças entre os fungicidas e a biomassa cítrica. $\mathrm{Na}$ análise de desfolha verificouse redução de $20 \%$ da AACPD comparando-se as formulações com e sem desfolha (Tabela 2).

$\mathrm{Na}$ avaliação de soma bruta final (SBfinal)

Tabela 1 - Valores de F calculados para produtos, desfolha e interação entre os fatores para as variáveis área abaixo da curva de progresso da doença (AACPD) e soma bruta final (SBfinal) da sigatoka amarela (Mycosphaerella musicola) na bananeira 'Prata' condições de campo em Agosto-Novembro/2007.

Table 1 - Values of $F$ calculated for products, defoliation and interaction among the factors for the variables area under disease progress curve (AUDPC) and final gross sum (SBfinal) of yellow sigatoka (Mycosphaerella musicola) for banana in field conditions of August to November / 2007.

\begin{tabular}{lcc}
\hline & \multicolumn{2}{c}{ Variáveis } \\
\cline { 2 - 3 } Fatores & AACPD & SBfinal \\
\hline Formulações & $3,71^{*}$ & $10,06^{* *}$ \\
Desfolha & $0,89^{\text {ns }}$ & $0,48^{\mathrm{ns}}$ \\
Interação formulações x desfolha & $1,57^{\mathrm{ns}}$ & $1,40^{\mathrm{ns}}$ \\
\hline
\end{tabular}

ns_ não significativo, ${ }^{*}$ - significativo ao nível de $5 \%$ de probabilidade $\mathrm{e}^{* *}$ - significativo ao nível de $1 \%$ de probabilidade pelo teste de F. 
Tabela 2 - Efeitos das formulações e da desfolha sanitária no desenvolvimento da sigatoka amarela (Mycosphaerella musicola) quantificado pela área abaixo da curva de progresso da doença e soma bruta final na bananeira 'Prata' sob condições de campo em Agosto-Novembro/2007.

Table 2 - Effect of formulations and defoliation in the development of yellow sigatoka (Mycosphaerella musicola) quantified by the area under disease progress curve and total gross ultimate in banana under field conditions in the period of August-November / 2007.

\begin{tabular}{|c|c|c|c|c|c|c|c|c|}
\hline \multirow{3}{*}{$\begin{array}{l}\text { Produto } \\
\text { Testemunha }\end{array}$} & \multicolumn{4}{|c|}{$\mathrm{AACPD}^{12}$} & \multicolumn{4}{|c|}{ SBfinal $^{1}$} \\
\hline & \multirow{2}{*}{$\begin{array}{r}\text { s/ desf. }{ }^{3} \\
10584\end{array}$} & \multirow{2}{*}{$\begin{array}{c}\text { c/desf. }{ }^{3} \\
7928\end{array}$} & \multicolumn{2}{|c|}{ Média } & \multirow{2}{*}{$\begin{array}{c}\text { s/ desf. }{ }^{3} \\
220\end{array}$} & \multirow{2}{*}{$\begin{array}{c}\text { c/desf. }{ }^{3} \\
104\end{array}$} & \multicolumn{2}{|c|}{ Média } \\
\hline & & & 9256 & A & & & 162 & A \\
\hline Biom. Cítrica ${ }^{4}$ & 2770 & 5496 & 4133 & B & 32 & 60 & 46 & B \\
\hline Fungicida $^{5}$ & 6338 & 2282 & 4310 & B & 28 & 12 & 20 & B \\
\hline Média & 6564 n.s. & 5235 & & & $93,3^{\text {n.s. }}$ & 58,5 & & \\
\hline \multicolumn{9}{|c|}{$\begin{array}{l}{ }^{1} \text { Média de cinco repetições. Médias separadas por diferentes médias são significativamente diferentes entre si pelo teste } \\
\text { de Duncan }(\mathrm{P}<5 \%) \text {. } \\
{ }^{2} \mathrm{AACPD}=\text { área abaixo da curva de progresso da doença calculada com base em seis datas de avaliações aos } 42,49,60 \text {, } \\
70,77 \text { e } 84 \text { dias após primeira pulverização. } \\
{ }^{3} \mathrm{~s} / \text { desf. = sem desfolha, c/ desf.= com desfolha. Tratamentos aplicados em intervalos de } 30 \text { dias. } \\
{ }^{4} \text { Biom. Cítrica= biomassa cítrica }\left(1,0 \mathrm{~L} \mathrm{ha}^{-1} \text { produto comercial). Tratamentos aplicados em intervalos de } 30 \text { dias. }\right. \\
{ }^{5} \text { Fungicidas }=\text { Tebuconazole }\left(0,5 \mathrm{~L} \mathrm{ha}^{-1}\right) \text { - tiofanato metílico }\left(0,6 \mathrm{~L} \mathrm{ha}^{-1}\right) \text { aplicados alternadamente em intervalos de } 30 \\
\text { dias. }\end{array}$} \\
\hline
\end{tabular}

verificou-se que todos os tratamentos diferenciaramse entre si para as formulações, mas não para desfolha (Tabela 1). A interação entre os fatores de formulações e desfolha também não foi significativa. Os fungicidas e a biomassa cítrica diferiram da testemunha, mas foram similares entre si. A biomassa cítrica e os fungicidas reduziram a variável em $73 \%$ e $83 \%$, respectivamente, em comparação com a testemunha (Tabela 2). O efeito da desfolha reduziu a SBfinal em $38 \%$ comparando-se os produtos com e sem desfolha, mas sem apresentar diferenças significativas.

A análise da curva de progresso da doença denota os maiores valores da doença para a testemunha em relação aos outros tratamentos na maioria das avaliações (Figura 1). Também se pode observar o efeito marcante dos produtos no desenvolvimento da doença. No tratamento dos fungicidas sem desfolha, por exemplo, se verificou uma grande redução dos valores dos 42 dias para 84 dias em razão da aplicação dos fungicidas sistêmicos. Pela figura podese verificar que o controle da doença pela biomassa cítrica foi positivo, pois reduziram significativamente as duas variáveis em comparação com a testemunha.

$\mathrm{O}$ número de pulverizações para controle sigatoka amarela varia de duas a oito por ciclo da cultura em diversos países (GANRY 2008), fato semelhante ao verificado no litoral catarinense. Todavia, existe uma tendência de aumento das aplicações em virtude da presença da sigatoka negra, o que aumenta a importância dos testes com diferentes fungicidas. O tratamento testado com tiofanato metílico e tebuconazole mostrou-se eficiente e segue as premissas de manejo da resistência. Estes fungicidas são utilizados em diferentes países para controle da sigatoka em programas de rotação de fungicidas (VICENTE 2006). Os fungicidas testados pertencem a diferentes grupos químicos, reduzindo o risco de aparecimento de populações resistentes do patógeno, conforme já registrado para sigatoka negra em outros países (LAPEYRE DE BELLAIRE et al. 2010).

Neste trabalho foram verificados resultados promissores para biomassa cítrica no controle da doença. Esta formulação foi testada com resultados promissores para os patógenos Mycosphaerella fijiensis, Plasmopara viticola e Hemileia vastatrix (HANADA et al. 2004, GALVÃO et al. 2006, RESENDE et al. 2006). Em testes in vitro foi verificada a total inibição da germinação de conídios de $M$. fijiensis na dose de $100 \mathrm{mg} \mathrm{L}^{-1}$. Efeito sanitizante similar foi verificado em frutos de banana na mesma dose (HANADA et al. 2004).

$\mathrm{Na}$ análise das variáveis verificou-se que os tratamentos com desfolha não apresentaram diferenças significativas em comparação aos sem desfolha. Contudo, esta prática tem destacada importância na 


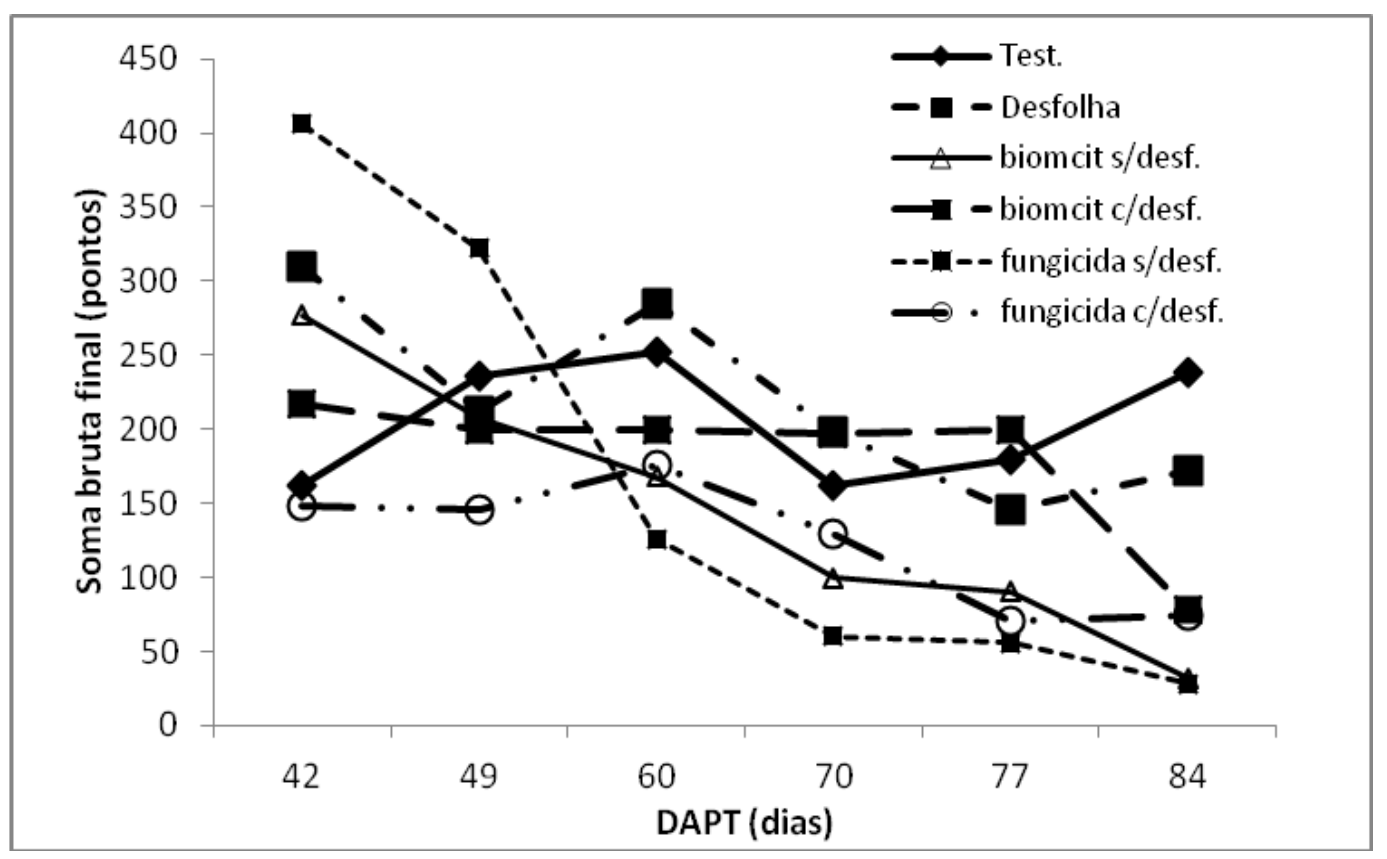

Figura 1 - Curvas de progresso da sigatoka amarela (Mycosphaerella musicola) sob tratamentos químicos e culturais em bananeira 'Prata' nas condições de campo em 2007. Tratamentos aplicados em intervalos de 30 dias. Test.=testemunha, Desfolha=somente desfolha, biomcit. s/ desf.=biomassa cítrica sem desfolha, biomcit. c/desf.=biomassa cítrica com desfolha, fungicida s/ desf.=fungicidas sem desfolha, fungucida $\mathrm{c} /$ desf=fungicidas com desfolha.

Figure 1 - Yellow sigatoka progress curves (Mycosphaerella musicola) in chemical and cultural control in banana" Prata" under field conditions in 2007. Treatments applied every 30 days. Test. $=$ Witness, defoliaton= only defoliation, biomcit. s / defoliation = citric biomass without defoliation, biomcit . $c /$ defoliation $=$ citric biomass with defoliation, fungicide $s /$ defoliation $=$ fungicides without defoliation, fung. $c /$ defoliation = fungicides with leaf defoliation.

redução de inoculo da doença devido à sobrevivência do patógeno nas folhas. Segundo STOVER (1971), M. musicola pode sobreviver por até oito semanas nas folhas, enquanto que $M$. fijiensis resiste por um período de até 20 semanas nas folhas (MARÍN 2003) e de 18 dias nas frutas (HANADA et al. 2004).

A eficiência da desfolha no controle da sigatoka negra foi comprovada experimentalmente por várias pesquisas (EMEBERI \& OBIEFUNA 1992, VARGAS et al. 2009). EMEBERI \& OBIEFUNA (1992) obtiveram controle de 10 a $18 \%$ pela remoção das folhas em diferentes densidades de plantio.

Novos trabalhos com freqüências de desfolha menores devem ser conduzidos para conhecer melhor seu efeito. Segundo BALBIN \& ZAPATA (2001), a desfolha semanal da bananeira somada a outras práticas resultou em melhor controle e retorno ao produtor. GUZMÁN \& VILLALTA (2006) obtiveram resultados satisfatórios com desfolhas semanais, enquanto neste trabalho a prática foi aplicada mensalmente. Segundo RODRIGUES et al. (2009), mantendo-se 10-12 folhas na floração foi suficiente para obterem-se boas produtividades e sanidade na Prata-anã.

\section{CONCLUSÕES}

Os fungicidas tiofanato metílico, tebucanazole e a biomassa cítrica nas doses testadas foram eficientes no controle da doença;

A desfolha mensal não foi eficiente no controle da sigatoka, mas deve ser estudada em diferentes freqüências de aplicação.

\section{REFERÊNCIAS}

AMIL AF et al. 2007. Dynamics of QoI sensitivity in Mycosphaerella fijiensis in Costa Rica during 2000 to 2003. Phytopathol. 97: 1451-1457.

ALVINDIA DG. 2012, Inhibitory influence of biocontrol agents, plant oils and an inorganic salt on Mycosphaerella fijiensis and Cordana musae, the causal pathogen of black 
sigatoka and leaf spot of banana. African J. Microbiol. Res. 6: 4179-4184.

BALBIN LAG \& ZAPATA JC. 2001. Integrated management of black and yellow sigatoka in plantain cv. Africa. Infomusa 10: 3-7.

BRENT KJ \& HOLLOMON DW. 2007. Fungicide resistance in crop pathogens: how it can be managed? Brussels: Croplife internacional. 50p.

BUREAU E. et al. 1992. El sistema de preaviso para el combate de la sigatoka negra en banana y platano. Panamá: UPEB. 40p.

CHILLET M et al. 2009. Sigatoka disease reduces greenlife of bananas. Crop Protec. 28: 41-45.

CORDEIRO ZJM et al. 2005. Doenças da bananeira. In: KIMATI H et al. Manual de Fitopatologia. São Paulo: Ceres p.99-118.

DONATO SLR et al. 2009. Comportamento fitotecnico da bananeira Prata-Anã e seus híbridos. Pesq. Agropec. Bras. 44: 1608-1615.

EMEBERI LC \& OBIEFUNA JC. 1992. Effects of leaf removal and intercropping on the incidence and severity of black Sigatoka disease at the establishment phase of plantains (Musa spp. AAB). Agric. Ecosyst. Environ. 39: 213-219.

ETEBU E \& YOUNG-HARRY W. 2011. Control of black sigatoka disease: challenges and prospects. African J. Agric. Res. 6: 508-514.

GALVÃO S et al. 2006. Avaliação da Eficiência de Produtos Alternativos para o Controle do Míldio e da Antracnose em Videiras (var. Niágara Branca). Agropec. Catarinense 19: 91-93.

GANRY J et al. 2008. A biological forecasting system to control sigatoka disease of bananas and plantains. Fruits 63: 381-387.

GUZMAN M \& VILLALTA R. 2006. Aporte de la deshoja sanitária y praticas adicionales em El control de la sigatoka negra em banano (Musa AAA). In: Manejo de la sigatoka negra em banano y plátano en America Latina y El Caribe. Resumenes... San Jose: Corbana-Inibap-Musalac. p.21.

HANADA R.E et al. 2004. Eficiência de desinfestantes na erradicação de conídios de Mycosphaerella fijiensis aderidos à superfícies de bananas. Fitopatol. Bras. 29: 9496.

JIMENEZ M et al. 2007. Organic banana production in Equador: its implication on black sigatoka development and plant soil nutritional status. Renew. Agric. Food Syst. 22: 297-302.

LAPEYRE DE BELLAIRE L et al. 2010. Black leaf streak is challenging the banana industry. Fruits 65: 327-342.

MARÍN DH et al. 2003. Black sigatoka: an increasing threat to banana cultivation. Plant Dis. 87: 208-222.

RESENDE MLV et al. 2006. Produtos indutores à base de bioindutores de resistência em plantas. Ver. Anual Patol. Plantas 14: 363-382.

RIBEIRO JÚNIOR JI. 2001. Análises estatísticas no
SAEG. Viçosa: UFV. 301p.

ROBINSON JC. 2006. Bananas and platains. Eastbourne: CAB internacional. 238p.

RODRIGUES MGV et al. 2009. Influência de diferentes níveis de desfolha na produção e qualidade dos frutos da bananeira Prata-Anã. Rev. Bras. Frut. 31: 755-762.

STOVER RH. 1971. Ascospore survival in Mycosphaerella musicola. Phytopathol. 61: 139-141.

SÔNEGO M et al. 2003. A fruticultura do litoral sul de Santa Catarina. Agropec. Catarinense 16: 26-32.

VAWDREY LL \& GRICE K. 2005. Evaluación en campo de las strobirulinas, triazoles y acibenzolar para el control de la sigatoka amarilla en Australia. Infomusa 14: 11-15.

VARGAS A et al. 2009. Effect of leaf pruning at flower emergence of banana plants (AAA) on fruit yield and black sigatoka (Mycosphaerella fijiensis) disease. Int. J. Pest Manag. 55: 19-25.

VICENTE LP. 2006. Manejo convencional y alternativo de la sigatoka negra em bananos: estado actual y perspectivas. Fitosanidad 10: 55-68. 